\title{
Effects of Lorentz Symmetry Breaking and scalar Potential on Relativistic Quantum Oscillator
}

\author{
Faizuddin Ahmed ${ }^{1}$ \\ National Academy Gauripur, Assam, 783331, India
}

\begin{abstract}
In this paper, we investigate the behaviour of a relativistic quantum oscillator under the effects of Lorentz symmetry violation determined by a tensor $\left(K_{F}\right)_{\mu \nu \alpha \beta}$ out of the Standard Model Extension. We analyze the quantum system under a Coulomb-type radial electric field and a uniform magnetic induced by Lorentz symmetry breaking effects under a Cornell-type potential, and obtain the bound states solution by solving the Klein-Gordon oscillator. We see a quantum effect due to the dependence of the angular frequency of the oscillator on the quantum numbers of the system, and the energy eigenvalues and the wave-function of the oscillator field get modified by the Lorentz symmetry breaking parameters as well as due to the presence of Cornell-type potential.
\end{abstract}

Keywords: Lorentz symmetry violation, Relativistic wave-equations: bound states solutions, scalar potential, electric \& magnetic field, biconfluent Heun's function .

PACS Number(s): 03.65.Pm, 11.30.Cp, 11.30.Qc

\section{Introduction}

The relativistic quantum dynamics of scalar particle by solving the KGoscillator in various space-times background has been investigated by many authors $[1,2,3,4,5,6,7,8,9,10,11]$. The Klein-Gordon oscillator [12]

\footnotetext{
${ }^{1}$ faizuddinahmed15@gmail.com ; faiz4U.enter@rediffmail.com
} 
was inspired by the Dirac oscillator [13] applied for spin- $\frac{1}{2}$ particle. In this work, we study the relativistic quantum oscillator in a possible scenario of anisotropy generated by Lorentz symmetry breaking term defined by a tensor $\left(K_{F}\right)_{\mu \nu \alpha \beta}$ that governs the Lorentz symmetry violation out of the Standard Model Extension. We consider the effects of a Coulomb-type potential induced by the Lorentz symmetry violation, and analyze the behaviour of a relativistic quantum oscillator by solving the Klein-Gordon oscillator subject to a scalar potential by modifying the mass term in the equation, and obtain the bound states solution.

The Lorentz symmetry is supposed to be a fundamental symmetry of physics. It contains two distinct sub-groups, rotation and boost. The Lorentz symmetry is a symmetry in low energy scale because of non-compactness of the Lorentz group that imposes an upper-bound for testing the Lorentz symmetry experimentally. There has been a great interest in theories endowed with Lorentz symmetry violation. This interest was initially motivated by the possibility of occurring this kind of violation in high energy theories defined at the Planck energy scale $[14,15,16,17,18,19,20]$. The Standard Model Extension (SME) $[20,21,22,23,24]$ is the theoretical effective structure that includes Lorentz-violating (LV) terms, generated as vacuum expectation values of tensors quantities, in the different sectors of the usual Standard Model. A large number of investigations in LV theories have been developed in recent years, addressing distinct sectors of the SME: fermion systems, the CPT-odd gauge sector, the CPT-even gauge sector (for references, see Ref. [25, 26, 27]).

The gauge sector of the Standard Model Extension possess two violating terms that modifies the transport properties of space-time since these terms break the Lorentz symmetry. These two terms are called the CPT-odd sector $[21,22]$ and the CPT-even sector $[28,29]$. The KG-equation under the effects of Lorentz symmetry violation $[21,22,30,31,32,33,34]$ is given by

$$
p^{\mu} p_{\mu} \Psi+\frac{\alpha}{4}\left(K_{F}\right)_{\mu \nu \alpha \beta} F^{\mu \nu}(x) F^{\alpha \beta}(x) \Psi=(M+S)^{2} \Psi,
$$

where $\alpha$ is a constant, $F_{\mu \nu}(x)=\partial_{\mu} A_{\nu}-\partial_{\nu} A_{\mu}$ is the electromagnetic field 
tensor, $\left(K_{F}\right)_{\mu \nu \alpha \beta}$ corresponds to a tensor that governs the Lorentz symmetry violation out of the Standard Model Extension, $M$ is the rest mass of the particle and $S$ is the scalar potential.

The Cornell-type scalar potential in cylindrical system $[5,35,36]$ includes two concepts-confinement and asymptotic freedom. The Coulomb-like part is responsible for the interactions at small distances whereas, and linear potential term is a long range that leads to the confinement phenomena of quarks. This type of potential has studied to obtain bound states solution of the wave-equations $[9,37,38,39,40,41]$, bound states of hadrons [42, 43], and the ground state of three quarks [44] in particle physics. This type of potential is given by given by

$$
S(r)=\eta_{L} r+\frac{\eta_{c}}{r}
$$

where $\eta_{L}>0, \eta_{c}>0$ are arbitrary constants.

The structure of this paper is as follows: in section $I I$, we introduce the Lorentz symmetry breaking effects defined by a tensor $\left(K_{F}\right)_{\mu \nu \alpha \beta}$ that governs the Lorentz symmetry violation out of the Standard Model Extension. Then, we analyse the behaviour of relativistic quantum oscillator by solving the KGoscillator under these effects of Lorentz symmetry breaking; in section III, we present our conclusions.

\section{Relativistic quantum oscillator under Lorentz Symmetry Breaking Effects}

We consider the Minkowski flat space-time

$$
d s^{2}=-d t^{2}+d r^{2}+r^{2} d \phi^{2}+d z^{2}
$$

where the ranges of the cylindrical coordinates are $-\infty<(t, z)<\infty, r \geq 0$ and $0 \leq \phi \leq 2 \pi$ 
For the geometry (3), the KG-equation under the effects of the Lorentz symmetry violation using (1) becomes

$$
\begin{aligned}
& {\left[-\frac{\partial^{2}}{\partial t^{2}}+\frac{1}{r} \frac{\partial}{\partial r}\left(r \frac{\partial}{\partial r}\right)+\frac{1}{r^{2}} \frac{\partial^{2}}{\partial \phi^{2}}+\frac{\partial^{2}}{\partial z^{2}}\right] \Psi+\frac{\alpha}{4}\left(K_{F}\right)_{\mu \nu \alpha \beta} F^{\mu \nu}(x) F^{\alpha \beta}(x) \Psi} \\
& =(M+S)^{2} \Psi
\end{aligned}
$$

One of the properties of the tensor $\left(K_{F}\right)_{\mu \nu \alpha \beta}$ is that it can be written in terms of $3 \times 3$ matrices

$$
\begin{aligned}
& \left(\kappa_{D E}\right)_{j k}=-2\left(K_{F}\right)_{0 j 0 k} \\
& \left(\kappa_{H B}\right)_{j k}=\frac{1}{2} \epsilon_{j p q} \epsilon_{k l m}\left(K_{F}\right)^{p q l m} \\
& \left(\kappa_{D B}\right)_{j k}=-\left(\kappa_{H E}\right)_{k j}=\epsilon_{k p q}\left(K_{F}\right)^{0 j p q}
\end{aligned}
$$

Note that the matrices $\left(\kappa_{D E}\right)_{j k}$ and $\left(\kappa_{H B}\right)_{j k}$ are symmetric that represent the parity-even sector of the tensor field $\left(K_{F}\right)_{\mu \nu \alpha \beta}$. On the other hand, the matrices $\left(\kappa_{D B}\right)_{j k}$ and $\left(\kappa_{H E}\right)_{k j}$ have no symmetry that represent the parityodd sector of the tensor field $\left(K_{F}\right)_{\mu \nu \alpha \beta}$. In this way, we can rewrite (4) in the form :

$$
\begin{aligned}
& {\left[-\frac{\partial^{2}}{\partial t^{2}}+\frac{\partial^{2}}{\partial r^{2}}+\frac{1}{r} \frac{\partial}{\partial r}+\frac{1}{r^{2}} \frac{\partial^{2}}{\partial \phi^{2}}+\frac{\partial^{2}}{\partial z^{2}}\right] \Psi } \\
+ & {\left[-\frac{\alpha}{2}\left(\kappa_{D E}\right)_{i j} E^{i} E^{j}+\frac{\alpha}{2}\left(\kappa_{H B}\right)_{j k} B^{i} B^{j}-\frac{\alpha}{2}\left(\kappa_{D B}\right)_{j k} E^{i} B^{j}\right] \Psi=(M+S)^{2} \Psi . }
\end{aligned}
$$

Let us consider a possible scenario of the Lorentz symmetry violation determined by the non-null terms $\left(\kappa_{D E}\right)_{11}=$ const, $\left(\kappa_{H B}\right)_{33}=$ const and $\left(\kappa_{D B}\right)_{13}=$ const and the field configuration is given by $[33,34]$ :

$$
\vec{B}=B_{0} \hat{z} \quad, \quad \vec{E}=\frac{\lambda}{r} \hat{r}
$$

where $B_{0}>0, \hat{z}$ is a unit vector in the $z$-direction, $\lambda$ is a constant associated with a linear distribution of electric charge along the axial direction, and $\hat{r}$ is the unit vector in the radial direction. 
To include the oscillator with the Klein-Gordon field, we change the following momentum operator $[1,2,3,4,5,6,7,8,9,10,11]$ :

$$
\vec{p} \rightarrow \vec{p}+i M \omega \vec{r}
$$

where $\omega$ is the oscillator frequency and $\vec{r}=r \hat{r}$ with $r$ being the distance from the particle to the axis. So we can write $\vec{p}^{2} \rightarrow(\vec{p}+i M \omega \vec{r})(\vec{p}-i M \omega \vec{r})$. Therefore, using the Lorentz symmetry violation term defined above with the field configuration (7) and finally using (8), equation (6) becomes

$$
\begin{aligned}
& {\left[-\frac{\partial^{2}}{\partial t^{2}}+\frac{1}{r}\left(\frac{\partial}{\partial r}+M \omega r\right)\left(r \frac{\partial}{\partial r}-M \omega r^{2}\right)+\frac{1}{r^{2}} \frac{\partial^{2}}{\partial \phi^{2}}+\frac{\partial^{2}}{\partial z^{2}}\right] \Psi} \\
& +\left[-\frac{\alpha}{2}\left(\kappa_{D E}\right)_{11} \frac{\lambda^{2}}{r^{2}}+\frac{\alpha}{2}\left(\kappa_{H B}\right)_{33} B_{0}^{2}-\frac{\alpha}{2} \frac{\lambda B_{0}}{r}\left(\kappa_{D B}\right)_{13}\right] \Psi=(M+S)^{2} \Psi .
\end{aligned}
$$

Since the metric is independent of time and symmetrical by translations along the $z$-axis, as well by rotations. It is reasonable to write the solution to Eq. (9) as

$$
\Psi(t, r, \phi, z)=e^{i(-E t+l \phi+k z)} \psi(r),
$$

where $E$ is the energy of the particle, $l=0, \pm 1, \pm 2, \ldots$ are the eigenvalues of the $z$-component of the angular momentum operator, and $k$ is a constant.

Therefore using the function (10) and scalar potential (2), we obtain the radial wave-equation for $\psi(r)$ :

$$
\psi^{\prime \prime}(r)+\frac{1}{r} \psi^{\prime}(r)+\left[\Lambda-\frac{j^{2}}{r^{2}}-\Omega^{2} r^{2}-\frac{b_{1}}{r}-b_{2} r\right] \psi(r)=0,
$$

where

$$
\begin{aligned}
& \Lambda=E^{2}-M^{2}-k^{2}-2 \eta_{L} \eta_{c}+\frac{1}{2} \alpha B_{0}^{2}\left(\kappa_{H B}\right)_{33}-2 M \omega \\
& j=\sqrt{l^{2}+\frac{1}{2} \alpha \lambda^{2}\left(\kappa_{D E}\right)_{11}+\eta_{c}^{2}} \\
& \Omega=\sqrt{M^{2} \omega^{2}+\eta_{L}^{2}} \\
& b_{1}=\alpha \lambda B_{0}\left(\kappa_{D B}\right)_{13}+2 M \eta_{c} \\
& b_{2}=2 M \eta_{L}
\end{aligned}
$$


Transforming $x=\sqrt{\Omega} r$ in the above equation (11), we have

$$
\left[\frac{d^{2}}{d x^{2}}+\frac{1}{x} \frac{d}{d x}+\zeta-x^{2}-\frac{j^{2}}{x^{2}}-\frac{\eta}{x}-\theta x\right] \psi(x)=0,
$$

where

$$
\zeta=\frac{\Lambda}{\Omega} \quad, \quad \eta=\frac{b_{1}}{\sqrt{\Omega}} \quad, \quad \theta=\frac{b_{2}}{\Omega^{\frac{3}{2}}} .
$$

Suppose the possible solution to the Eq. (13) is

$$
\psi(x)=x^{j} e^{-\frac{1}{2}(x+\theta) x} H(x) .
$$

Substituting the solution (15) into the Eq. (13), we obtain the following equation

$$
H^{\prime \prime}(x)+\left[\frac{1+2 j}{x}-2 x-\theta\right] H^{\prime}(x)+\left[-\frac{\beta}{x}+\Theta\right] H(x)=0,
$$

where

$$
\Theta=\zeta+\frac{\theta^{2}}{4}-2(1+j) \quad, \quad \beta=\eta+\frac{\theta}{2}(1+2 j)
$$

Equation (16) is the biconfluent Heun's differential equation $[1,2,4,5,6,7$, $8,9,35,45,46,47]$ with $H(x)$ is the Heun polynomials function.

The above equation (16) can be solved by the Frobenius method. Writing the solution as a power series expansion around the origin [48]:

$$
H(x)=\sum_{i=0}^{\infty} d_{i} x^{i}
$$

Substituting the power series solution into the Eq. (18), we obtain the following recurrence relation

$$
d_{n+2}=\frac{1}{(n+2)(n+2+2 j)}\left[\left(\eta+\theta\left(n+\frac{3}{2}+j\right)\right) d_{n+1}-(\Theta-2 n) d_{n}\right] .
$$

With few coefficients are

$$
\begin{aligned}
& d_{1}=\left(\frac{\eta}{1+2 j}+\frac{\theta}{2}\right) d_{0} \\
& d_{2}=\frac{1}{4(1+j)}\left[\left(\eta+\theta\left(j+\frac{3}{2}\right)\right) d_{1}-\Theta d_{0}\right] .
\end{aligned}
$$


The power series expansion $H(x)$ becomes a polynomial of degree $n$ by imposing the following two conditions $[1,2,4,5,6,7,8,9,11,35]$

$$
\begin{aligned}
\Theta & =2 n, \quad(n=1,2, \ldots) \\
d_{n+1} & =0 .
\end{aligned}
$$

By analyzing the first condition, we obtain following equation of the energy eigenvalue $E_{n, l}$ :

$$
\begin{aligned}
E_{n, l}^{2} & =M^{2}+k^{2}+2 \eta_{L} \eta_{c}+2\left[M \omega+\Omega\left(n+1+\sqrt{l^{2}+\frac{1}{2} \alpha \lambda^{2}\left(\kappa_{D E}\right)_{11}+\eta_{c}^{2}}\right)\right] \\
& -\frac{\alpha}{2} B_{0}^{2}\left(\kappa_{H B}\right)_{33}-\frac{M^{2} \eta_{L}^{2}}{\Omega^{2}} .
\end{aligned}
$$

It is worth mentioning that we have adjusted the parameters such that $\left(k^{2}+2 \eta_{L}\left(n+1+\eta_{c}+\sqrt{l^{2}+\frac{1}{2} \alpha \lambda^{2}\left(\kappa_{D E}\right)_{11}+\eta_{c}^{2}}\right)\right)>\frac{1}{2} \alpha B_{0}^{2}\left(\kappa_{H B}\right)_{33}+\frac{M^{2} \eta_{L}^{2}}{\Omega^{2}}$ and hence, the energy eigenvalue is real. Note that Eq. (22) is not the general expression of the relativistic energy eigenvalues of the oscillator field. One can obtain the individual energy levels and eigenfunctions one by one by imposing the additional recurrence condition $d_{n+1}=0$ on the eigenvalue problem.

The corresponding wave-functions are given by

$$
\psi_{n, l}(x)=x^{\sqrt{l^{2}+\frac{1}{2} \alpha \lambda^{2}\left(\kappa_{D E}\right)_{11}+\eta_{c}^{2}}} e^{-\frac{1}{2}\left[x+\frac{2 M}{\sqrt{\eta_{L}}}\right] x} H(x) .
$$

Now, we evaluate the individual energy levels and eigenfunctions one by one as in $[1,2,4,5,6,7,8,9,11,35]$. For example, $n=1$, we have $\Theta=2$ and $d_{2}=0$ which implies

$$
\begin{aligned}
& \Rightarrow \frac{2}{\eta+\theta\left(\frac{3}{2}+j\right)} d_{0}=\left(\frac{\eta}{1+2 j}+\frac{\theta}{2}\right) d_{0} \\
& \Rightarrow \Omega_{1, l}^{3}-\left[\frac{b_{1}^{2}}{2(1+2 j)}\right] \Omega_{1, l}^{2}-b_{1} b_{2}\left[\frac{1+j}{1+2 j}\right] \Omega_{1, l}-\frac{b_{2}^{2}}{8}(3+2 j)=0,
\end{aligned}
$$

a constraint on the parameter $\Omega_{1, l}$, that is, on the angular frequency of the oscillator $\omega_{1, l}$. We can see that the allowed values of the angular frequency 
of the oscillator $\omega$ depends on the quantum numbers $\{n, l\}$ of the relativistic system, and the parameters associated with the Lorentz Symmetry breaking effects. In particular, Eq. (24) is a cubic equation for the parameter $\Omega_{1, l}$ for the radial mode $n=1$, and there is a real solution of it which gives us the first degree polynomial to the function $H(x)$. Thus, the possible values of the angular frequency of the oscillator is given by

$$
\omega_{1, l}=\sqrt{\Omega_{1, l}^{2}-\eta_{L}^{2}} .
$$

Therefore, the ground state energy level for the radial mode $n=1$ is given by

$$
\begin{aligned}
E_{1, l}^{2} & =M^{2}+k^{2}+2 \eta_{L} \eta_{c}+2\left[M \omega_{1, l}+\Omega_{1, l}\left(2+\sqrt{l^{2}+\frac{1}{2} \alpha \lambda^{2}\left(\kappa_{D E}\right)_{11}+\eta_{c}^{2}}\right)\right] \\
& -\frac{\alpha}{2} B_{0}^{2}\left(\kappa_{H B}\right)_{33}-\frac{M^{2} \eta_{L}^{2}}{\Omega_{1, l}^{2}} .
\end{aligned}
$$

And the ground state eigenfunction is

$$
\psi_{1, l}(x)=x^{\sqrt{l^{2}+\frac{1}{2} \alpha \lambda^{2}\left(\kappa_{D E}\right)_{11}+\eta_{c}^{2}}} e^{-\frac{1}{2}\left[x+\frac{2 M \eta_{L}}{\Omega_{1, l}^{\frac{3}{2}}}\right] x}\left(1+d_{1} x\right)
$$

where we have chosen $d_{0}=1$ and

$$
d_{1}=\frac{1}{\sqrt{\Omega_{1, l}}}\left[\frac{\alpha \lambda B_{0}\left(\kappa_{D B}\right)_{13}+2 M \eta_{c}}{\left(1+\sqrt{l^{2}+\frac{1}{2} \alpha \lambda^{2}\left(\kappa_{D E}\right)_{11}+\eta_{c}^{2}}\right)}+\frac{2 M \eta_{L}}{\Omega_{1, l}}\right]
$$

We can see that the lowest energy state (26) plus the ground state wavefunction (27)-(28) along with the restriction on the angular frequency $\omega$ of the oscillator given by Eqs. (24)-(25) is defined for the radial mode $n=1$. This effect arises due to the presence of a Cornell-type potential, and the Lorentz symmetry breaking parameters $\left(\alpha, B_{0}, \lambda,\left(\kappa_{H B}\right)_{33},\left(\kappa_{D B}\right)_{13},\left(\kappa_{D E}\right)_{11}\right)$. Also we see that the energy eigenvalues and the corresponding wave-function get modified due to the presence of these parameters in the quantum system. 


\section{Special case corresponds to $\left(\kappa_{H B}\right)_{33}=0$, and $\left(\kappa_{D E}\right)_{11}=0$}

Here we interest to study a relativistic scalar particle under the Lorentz symmetry breaking effects with one non-null components $\left(\kappa_{D B}\right)_{13}=$ const $=$ $\kappa$ of the tensor $\left(K_{F}\right)_{\mu \nu \alpha \beta}$ that governs the Lorentz symmetry violation of the Standard Model Extension.

The radial wave-equation (11) becomes

$$
\psi^{\prime \prime}(r)+\frac{1}{r} \psi^{\prime}(r)+\left[\Xi-\frac{\tau^{2}}{r^{2}}-\Omega^{2} r^{2}-\frac{b_{1}}{r}-b_{2} r\right] \psi(r)=0,
$$

where $\Omega, b_{1}, b_{2}$ are given earlier and

$$
\begin{aligned}
& \Xi=E^{2}-M^{2}-k^{2}-2 \eta_{L} \eta_{c}-2 M \omega \\
& \tau=\sqrt{l^{2}+\eta_{c}^{2}}
\end{aligned}
$$

Transforming $x=\sqrt{\Omega} r$ in the above equation (29), we have

$$
\left[\frac{d^{2}}{d x^{2}}+\frac{1}{x} \frac{d}{d x}+\Pi-x^{2}-\frac{\tau^{2}}{x^{2}}-\frac{\eta}{x}-\theta x\right] \psi(x)=0
$$

where $\Pi=\frac{\Xi}{\Omega}$.

Suppose the possible solution to the Eq. (13) is

$$
\psi(x)=x^{\tau} e^{-\frac{1}{2}(x+\theta) x} H(x)
$$

Substituting the solution (32) into the Eq. (31), we obtain the following equation

$$
H^{\prime \prime}(x)+\left[\frac{1+2 \tau}{x}-2 x-\theta\right] H^{\prime}(x)+\left[-\frac{\Upsilon}{x}+\Delta\right] H(x)=0,
$$

where

$$
\Delta=\Pi+\frac{\theta^{2}}{4}-2(1+\tau) \quad, \quad \Upsilon=\eta+\frac{\theta}{2}(1+2 \tau) .
$$

Equation (33) is the biconfluent Heun's differential equation $[1,2,4,5,6,7$, $8,9,35,45,46,47]$ with $H(x)$ is the Heun polynomials function. 
Substituting the power series solution (19) into the equation (33), we obtain the following recurrence relation

$$
d_{n+2}=\frac{1}{(n+2)(n+2+2 \tau)}\left[\left(\eta+\theta\left(n+\frac{3}{2}+\tau\right)\right) d_{n+1}-(\Delta-2 n) d_{n}\right] .
$$

With few coefficients are

$$
\begin{aligned}
& d_{1}=\left(\frac{\eta}{1+2 \tau}+\frac{\theta}{2}\right) d_{0}, \\
& d_{2}=\frac{1}{4(1+\tau)}\left[\left(\eta+\theta\left(\tau+\frac{3}{2}\right)\right) d_{1}-\Delta d_{0}\right] .
\end{aligned}
$$

The power series expansion $H(x)$ becomes a polynomial of degree $n$ by imposing the following two conditions [1, 2, 4, 5, 6, 7, 8, 9, 11, 35]

$$
\begin{aligned}
\Delta & =2 n, \quad(n=1,2, \ldots) \\
d_{n+1} & =0 .
\end{aligned}
$$

By analysing the first condition $\Delta=2 n$, we obtain the energy eigenvalues $E_{n, l}$ expression:

$E_{n, l}= \pm \sqrt{M^{2}+k^{2}+2 \eta_{L} \eta_{c}+2\left[M \omega+\Omega\left(n+1+\sqrt{l^{2}+\eta_{c}^{2}}\right)\right]-\frac{M^{2} \eta_{L}^{2}}{\Omega^{2}}}$

And the radial wave-function is given by

$$
\psi_{n, l}(x)=x^{\sqrt{l^{2}+\eta_{c}^{2}}} e^{-\frac{1}{2}\left[x+\frac{2 M \eta_{L}}{\Omega^{\frac{3}{2}}}\right] x} H(x) .
$$

Following the similar technique as done above, one can obtain the individual energy level and wave-function. We have obtained the lowest state energy level and the eigenfunction as

$$
\begin{aligned}
& E_{1, l}= \pm \sqrt{M^{2}+k^{2}+2 \eta_{L} \eta_{c}+2\left[M \omega_{1, l}+\Omega_{1, l}\left(2+\sqrt{l^{2}+\eta_{c}^{2}}\right)\right]-\frac{M^{2} \eta_{L}^{2}}{\Omega_{1, l}^{2}}}, \\
& \psi_{1, l}(x)=x^{\sqrt{l^{2}+\eta_{c}^{2}}} e^{-\frac{1}{2}\left[x+\frac{2 M \eta_{L}}{\Omega_{1, l}^{\frac{3}{2}}}\right] x}\left(1+d_{1} x\right)
\end{aligned}
$$


where we have chosen $d_{0}=1$ and

$$
d_{1}=\frac{1}{\sqrt{\Omega_{1, l}}}\left[\frac{\alpha \lambda B_{0} \kappa+2 M \eta_{c}}{\left(1+2 \sqrt{l^{2}+\eta_{c}^{2}}\right)}+\frac{2 M \eta_{L}}{\Omega_{1, l}}\right]
$$

with the following constraints on the angular frequency $\omega$ of the oscillator

$$
\begin{aligned}
& \Omega_{1, l}^{3}-\left[\frac{b_{1}^{2}}{2(1+2 \tau)}\right] \Omega_{1, l}^{2}-b_{1} b_{2}\left[\frac{1+\tau}{1+2 \tau}\right] \Omega_{1, l}-\frac{b_{2}^{2}}{8}(3+2 \tau)=0, \\
& \omega_{1, l}=\frac{1}{M} \sqrt{\Omega_{1, l}^{2}-\eta_{L}^{2}}
\end{aligned}
$$

Here also we see that the energy eigenvalues and the corresponding wavefunction get modified due to the presence of a Cornell-type potential, and the Lorentz symmetry breaking parameters $\left(\alpha, B_{0}, \lambda,\left(\kappa_{D B}\right)_{13}\right)$ in the quantum system.

\section{Conclusions}

In this work, we have relaxed the renormalization property of our model because of tiny values contribute by Lorentz symmetry breaking terms that go for energy scales beyond the Standard Model. The scenario of Lorentz symmetry violation (LSV) is defined by a Coulomb-type radial electric field produced by an electric charge distribution, a constant magnetic along zdirection, and the tensor background that governs the Lorentz symmetry breaking effects possessing the non-null terms $\left\{\left(\kappa_{D B}\right)_{13},\left(\kappa_{D E}\right)_{11},\left(\kappa_{H B}\right)_{33}\right\}$. To study the behaviour of the Klein-Gordon oscillator field in the LSV under a scalar potential, we have introduced this scalar potential as a modification of the mass term $M \rightarrow M+S(r)$ in the Klein-Gordon oscillator, and derived the radial wave-equation. For a suitable wave-function, the biconfluent Heun differential equation form is arrived. Using the power series solution into the Heun equation, we see that the power series solution becomes a polynomial of degree $n$ by imposing two conditions simultaneously. Using the first condition $\Theta=2 n$, the non-compact expression of the energy eigenvalues $E_{n, l}$ Eq. 
(22) is obtained. Imposing the second condition $d_{n+1}=0$, one can obtain the individual energy level $E_{1, l}, E_{2, l}, \ldots$ and the wave-function $\psi_{1, l}, \psi_{2, l}, \ldots$ one by one. As for example, we have obtained the lowest state energy level Eq. (26) and the corresponding wave-function Eqs. (27)-(28) along with the restriction (25) imposed on the angular frequency $\omega_{1, l}$ of the oscillator that gives us the allowed values for the radial mode $n=1$. By similar technique, one can obtain a different relation of the oscillator frequency $\omega_{2, l}$ for the radial mode $n=2$ and so on. Thus, we have seen that the angular frequency $\omega_{n, l}$ of the oscillator depends on quantum numbers $\{n, l\}$ of the system which shows a quantum effect, and on the Lorentz symmetry breaking parameters $\left\{\alpha, \lambda, B_{0},\left(\kappa_{D E}\right)_{11},\left(\kappa_{D B}\right)_{13}\right\}$. We have also seen that the presence of various non-null components of the tensor that governs the Lorentz symmetry breaking effects, and the Cornell-type potential modified the energy spectrum and the wave-function of the oscillator field.

\section{Conflict of Interest}

There is no conflict of interest regarding publication this paper.

\section{Data Availability}

No data has been used to prepare this paper.

\section{References}

[1] F. Ahmed, Eur. Phys. J. C 78, 598 (2018).

[2] F. Ahmed, Adv. High Energy Phys. 2020, 5691025 (2020).

[3] A. Boumali and N. Messai, Can. J. Phys. 92, 1460 (2014). 
[4] J. Carvalho, A. M. M. Carvalho, E. Cavalcante, and C. Furtado, Eur. Phys. J. C 76, 365 (2016).

[5] Z. Wang, Z. Long, C. Long and M. Wu, Eur Phys. J. Plus 130, 36 (2015).

[6] M. Hosseinpour, H. Hassanabadi and Marc de Montigny, Int. J. Geom. Meths Mod. Phys. 15 (10), 1850165 (2018).

[7] F. Ahmed, Int. J. Geom. Meths Mod. Phys. 17 (09), 2050138 (2020).

[8] B. -Q. Wang, Z. -W. Long, C. -Y. Long and S. -R. Wu, Mod. Phys. Lett. A 33, 1850025 (2018).

[9] M. Hosseini, H. Hassanabadi, S. Hassanabadi and P. Sedaghatnia, Int. J. Geom. Meths. Mod. Phys. 16, 1950054 (2019).

[10] H. Sobhani, H. Hassanabadi and W. S. Chung, Int. J. Geom. Meths Mod. Phys. 15 (03), 1850037 (2018).

[11] F. Ahmed, EPL 130, 40003 (2020).

[12] S. Bruce and P. Minning, II Nuovo Cimento A 106, 711 (1993).

[13] M. Moshinsky and A. Szczepaniak, J. Phys. A : Math. Gen. 22, L817 (1989).

[14] V. A. Kostelecky and S. Samuel, Phys. Rev. Lett. 63 (1989) 224.

[15] V. A. Kostelecky and S. Samuel, Phys. Rev. Lett. 66 (1991) 1811.

[16] V. A. Kostelecky and S. Samuel, Phys. Rev. D 39 (1989) 683.

[17] V. A. Kostelecky and S. Samuel, Phys. Rev. D 40 (1989) 1886.

[18] V. A. Kostelecky and R. Potting, Nucl. Phys. B 359 (1991) 545. 
[19] V. A. Kostelecky and R. Potting, Phys. Rev. D 51 (1995) 3923.

[20] V. A. Kostelecky and R. Potting, Phys. Lett. B 381 (1996) 89.

[21] D. Colladay and V. A. Kostelecky, Phys. Rev. D 55, 6760 (1997).

[22] D. Colladay and V.A. Kostelecky, Phys. Rev. D 58, 116002 (1998).

[23] S. R. Coleman and S. L. Glashow, Phys. Rev. D 59 (1999) 116008.

[24] L. Campanelli and P. Cea, Physica B 359 (1991).

[25] R. Casana, M. M. Ferreira Jr., R. V. Maluf and F. E. P. dos Santos, Phys. Lett. B 726 (2013) 815.

[26] V. A. Kostelecky and N. Russell, Rev. Mod. Phys. 83 (2011) 11.

[27] J. A. de Sales, T. Costa-Soares and V. J. Vasquez Otoya, Physica A 391 (2012) 5422 .

[28] S. Carroll, G. Field and R. Jackiw, Phys. Rev. D 41, 1231 (1990).

[29] A. P. Baeta Scarpelli, H. Belich, J. L. Boldo, L. P. Colatto, J. A. HelayelNeto, A. L. M. A. Nogueira, Nucl. Phys. Proc. Suppl. 127, 105 (2004).

[30] V. A. Kostelecky and M. Mewes, Phys. Rev. Lett. 87, 251304 (2001).

[31] V. A. Kostelecky and M. Mewes, Phys. Rev. D 66, 056005 (2002).

[32] H. Belich, F. J. L. Leal, H. L. C. Louzada and M. T. D. Orlando, Phys. Rev. D 86, 125037 (2012).

[33] K. Bakke and H. Belich, Ann. Phys. (N. Y.) 360, 596 (2015).

[34] R. L. L. Vitoria, H. Belich and K. Bakke, Eur. Phys. J. Plus (2017) 132 : 25 . 
[35] E. R. Figueiredo Medeiros, E. R. Bezerra de Mello, Eur. Phys. J. C 72, 2051 (2012).

[36] M. K. Bahar and F. Yasuk, Adv. High Energy Phys. 2013, 814985 (2013).

[37] M. de Montigny, M. Hosseinpour and H. Hassanabadi, Int. J. Mod. Phys. A 31, 1650191 (2016).

[38] M. S. Cunha, C. R. Muniz, H. R. Christiansen and V. B. Bezerra, Eur. Phys. J. C 76, 512 (2016).

[39] F. Ahmed, Adv. High Energy Phys. 2020, 4832010 (2020).

[40] H. Hassanabadi, E. Maghsoodi and S. Zarrinkamar, Ann. Phys. (Berlin) 525, 944 (2013).

[41] M. Hosseinpour, H. Hassanabadi and Marc de Montigny, Int. J. Geom. Meths Mod. Phys. 15, 1850165 (2018).

[42] H. Hassanabadi and S. Rahmani, Few-Body Syst. 56, 691 (2015).

[43] H. Hassanabadi, S. Rahmani and S. Zarrinkamar, Phys. Rev. D 90, 074024 (2014).

[44] C. Alexandrou, P. de Forcrand and O. Jahn, Nuclear Phys. B (Proc. Supp.) 119, 667 (2003).

[45] A. Ronveaux, Heun's Differential Equations, Oxford University Press, Oxford ( 1995).

[46] S. Y. Slavyanov and W. Lay, Special Functions: A Unified Theory Based in Singularities, Oxford University Press, New York (2000).

[47] M. Abramowitz and I. A. Stegum, Hand book of Mathematical Functions, Dover Publications Inc., New York (1965). 
[48] G. B. Arfken and H. J. Weber, Mathematical Methods For Physicists, Elsevier Academic Press, London (2005). 